\title{
EVIDENCIAS DE GLACIACION EN EL VALLE DEL GENERAL (COSTA RICA) DURANTE EL PLEISTOCENO TARDIO
}

\author{
Roberto Protti \\ Geotest, Apdo. 1291 - 2050 San Pedro, San José, Costa Rica
}

(Recibido 27/3/1997; Aceptado 4/7/1997)

\begin{abstract}
Glaciers and glacier morphology have been reported in Costa Rica only in the highest peaks of the Talamanca Range, over $3000 \mathrm{~m}$. However, this paper reports the existence of glacier morphology at elevations of less than $1000 \mathrm{~m}$ in Valle del General, specifically along the western flank of the Talamanca Range.

"U" shape valleys, frontal and lateral moraines, megalithic deposits with striation and glacial markings, polished rock planes, striae and groove cuts parallel to valleys are some of the evidence found in the San Rafael, San Pedro, and Peñas Blancas rivers. These features indicate the presence of glaciation during the Late Pleistocene in the Valle del General in Costa Rica.
\end{abstract}

RESUMEN: La presencia de glaciares y relieves producto del modelado glaciar ha sido descrita para Costa Rica únicamente en las partes más altas de la Cordillera de Talamanca, a elevaciones superiores a los $3000 \mathrm{~m}$ sobre el nivel del mar. Sin embargo, en este trabajo se reporta la existencia de relieves originados por la acción de glaciares a elevaciones menores que 1000 metros sobre el nivel del mar en el Valle del General, específicamente en el flanco occidental de la cordillera de Talamanca.

Valles en "U", depósitos de morrenas laterales y frontales, depósitos de megalitos estriados y acanalados, superficies de roca pulidas con texturas "aborregadas", estrías y acanaladuras paralelas a los valles, son algunas de las evidencias encontradas en las cuencas medias de los ríos San Rafael, San Pedro y Peñas Blancas, que indican la ocurrencia de glaciación durante el período Pleistoceno tardío en el Valle del General en Costa Rica.

\section{INTRODUCCIÓN}

Weyl (1957), describió por primera vez la presencia de vestigios de morfologías de origen glaciar en las cumbres de la Cordillera de Talamanca, en el sector sur-central de Costa Rica. En el área del Parque Nacional de Chirripó se aprecian morfologías típicas de modelado glacial, tales como amplios valles con sección transversal en "U", disectados en forma de valles en "V" por la erosión fluvial actual, extensas superfices de roca pulimentada con estrías y acanaladuras producidas por el desplazamiento de masas de hielo sobre ellas, y depósitos de morrenas con megalitos angulosos de caras planas pulidas.
Mora (1979), en sus descripciones de la Formación El General, menciona la presencia de fanglomerados con bloques de hasta $4 \mathrm{~m}$ en los depósitos de la región del "piedemonte" de la cordillera de Talamanca, descritos como abanicos producto de la erosión masiva de la cordillera tanto durante su emplazamiento como durante el deshielo de los glaciares del Pleistoceno.

De las referencias citadas, se desprende que las descripciones de morfologías de origen glaciar, hasta la fecha, se limitan a la morfología de las cumbres de la Cordillera de Talamanca, sobre la elevación actual de 3000 m.s.n.m., y a los depósitos de abanicos en la zona de "piedemonte", originados durante el deshielo de los 
glaciares. Sin embargo, durante los primeros meses del año 1997, se han identificado tres valles tributarios del río General, ubicados entre las elevaciones 700 y 1300 m.s.n.m, en los que existen evidencias de que algunos glaciares del Pleistoceno alcanzaron niveles más bajos que los reportados en la cordillera. Las tres cuencas en donde se ha identificado este tipo de morfología son las cuencas medias de los ríos Peñas Blancas, San Pedro, y San Rafael (Fig. 1).

En este trabajo se describe las morfologías de origen glaciar localizadas en las cuencas mencionadas, sin embargo, es posible que existan otras evidencias, principalmente en las cuencas

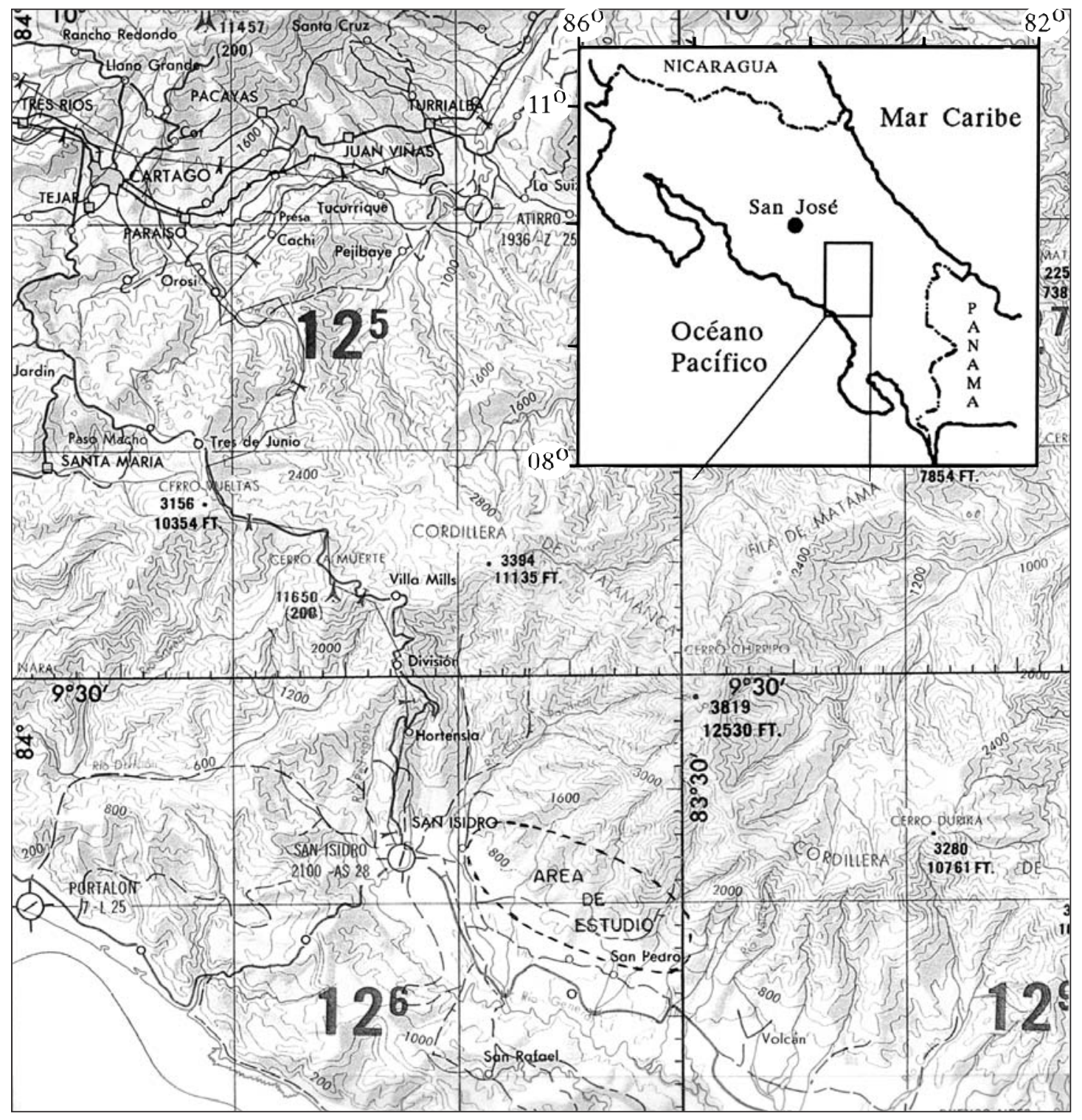

Fig. 1: Mapa de ubicación de la zona estudiada. Se trata del flanco suroeste de la cordillera de Talamanca, en el sector del macizo del Chirripó y comprende las cuencas de los rios Peñas Blancas, San Pedro y San Rafael. 
tributarias del río General ubicadas hacia el este de la región estudiada. Asimismo, se analizan las relaciones entre la edad relativa de los abanicos de pie de monte y los eventos glaciares de finales del período Pleistoceno.

\section{CONTEXTO GEOLÓGICO}

La región del "piedemonte" de la Cordillera de Talamanca, en el sector del Valle del General, está compuesta por un basamento de rocas sedimentarias de la Formación Térraba de edad Oligoceno-Mioceno (Sprechman, 1984), en donde ocurren litologías que varían desde conglomerados varicoloreados hasta secuencias de areniscas y lutitas tipo turbidita, las que se observan particularmente en la cuenca del río Peñas Blancas.

Las rocas del basamento sedimentario se encuentran cubiertas e intercaladas con gruesos derrames volcánicos de composición basáltica, tal como se observa actualmente en las cuencas medias de los rios San Rafael y San Pedro. Estas rocas se encuentran intruidas por granitos y granodioritas de edad Mioceno (Sprechman, 1984), contemporáneos con el levantamiento de la cordillera. Se ha observado intrusiones de granodioritia en forma de diques tanto dentro de las rocas sedimentarias, como dentro de los basaltos en la cuenca del río San Pedro.

La estructura geológica general de esta parte de la cordillera, es la de un monoclinal intruido buzando hacia el noreste.

Hacia la zona del piedemonte de la cordillera ocurren extensos y gruesos abanicos de depósitos no consolidados, sobre los que se emplazaron las lenguas glaciares que descendieron desde la cordillera hasta el Valle del General.

\section{MORFOLOGÍAS DE ORIGEN GLACIAR}

En la figura 2, se muestra la ubicación de los sitios en donde se han localizado morfologías de origen glaciar. Algunos de estos valles muestran un amplio perfil transversal en "U" dentro del que la erosión fluvial actual ha producido pequeñoas valles con sección en "V" (Foto 1).

Dentro de los valles de los ríos San Pedro y San Rafael existen depósitos de bloques megaliticos con tamaños que alcanzan los $15 \mathrm{~m}$. Estos depósitos, contrastan con la granulometría de los bancos aluviales actuales, de manera que se presenta una notable bimodalidad en la granulometría de los depósitos ubicados en el lecho de estos ríos y en sus terrazas laterales. Los depósitos aluviales actuales muestran bloques redondeados con tamaño máximo de $1 \mathrm{~m}$, con una gradación normal hacia grava y arena, mientras que en los depósitos glaciares se encuentran bloques de 10 a $15 \mathrm{~m}$ de diámetro equivalente, con distribución granulométrica errática, angulosos, con amplias caras planas pulidas y con estrías y acanaladuras propias de la erosión producida por el desplazamiento de grandes masas de hielo sobre la roca. Detalles de este tipo de depósitos y de los bloques megalíticos que los componen, se muestran en las fotografías 3 a 11.

Dentro de la cuenca del río San Rafael, a unos $2 \mathrm{~km}$ al norte de la localidad de San Jerónimo, y a la elevación de 1300 m.s.n.m., se observa sobre la margen izquierda del río una amplia superficie de roca pulimentada (Fotos 2 y 8), con acanaladuras y estrías paralelas al cauce actual del río. Esta parte de la cuenca, se ubica dentro de una morfología de tipo "artesa", posiblemente en el sitio de intersección de dos cursos glaciares que descendían de la cordillera dentro de los cauces actuales de los rios San Rafael y Zapotal (Fig. 2, Foto 5).

En la zona en la que los valles de los ríos San Pedro y San Rafael alcanzan la llanura de piedemonte del Valle del General, se encuentran depósitos megalíticos con características de morrenas, tanto laterales como frontales. Aproximadamente a $1 \mathrm{~km}$ aguas arriba de la confluencia entre la quebrada Peregrina y el río Unión, se observa uno de estos depósitos de morrenas laterales, que se extiende hasta la zona de la localidad de Laguna (Fig. 2, Foto 3), en donde se observan bloques rocosos con dimensiones megalíticas, asociados a una posible morrena frontal. Esta morrena se ubica a una elevación actual de 860 m.s.n.m, y su ubicación marca la posición del frente de avance máximo de los glaciares. Como se deduce de esta observación, estos glaciares alcanzaron elevaciones menores que los 1000 m.s.n.m. dentro del Valle del General actual.

Una de las estructuras más notables de morfología glaciar que permite la datación relativa de los eventos geológicos del Pleistoceno, se localiza en el área en que el valle del río San Pedro alcanza la región de abanicos de piedemonte. Se trata de un amplio valle en "U", con una ancho de $500 \mathrm{~m}$, que se encuentra emplazado sobre un grueso y extenso 


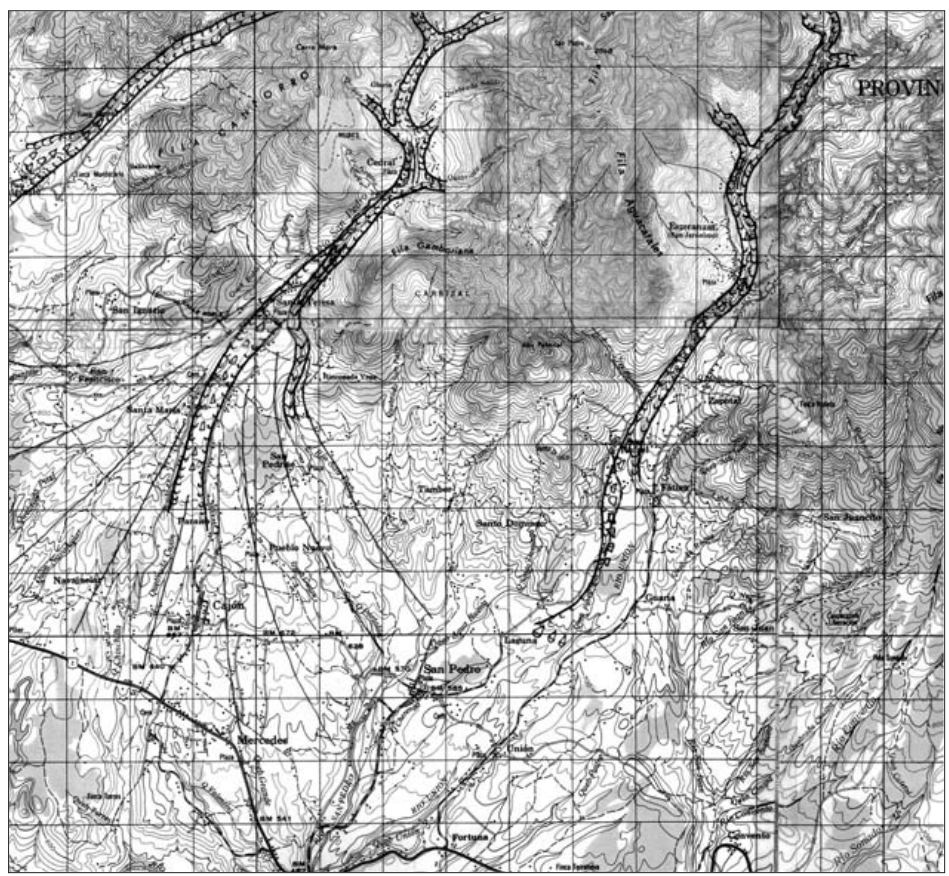

\section{SIMBOLOGIA \\ Cauce Glacial en "U"

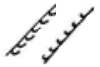 \\ Acanaladuras y estrias \\ III \\ Morrena frontal \\ $\theta A$ \\ Morrena lateral \\ Morena de fondo \\ $\Delta \Delta \Delta$ \\ Cauce fluvial en "V" \\ Abanico aluvial-periglacial \\ Y}

Fuente: hojas San Isidro, Durika, Buenos Aires y Repunta Escala 1:50 000 I:G:N:

Fig. 2: Mapa geomorfológico del área de estudio.

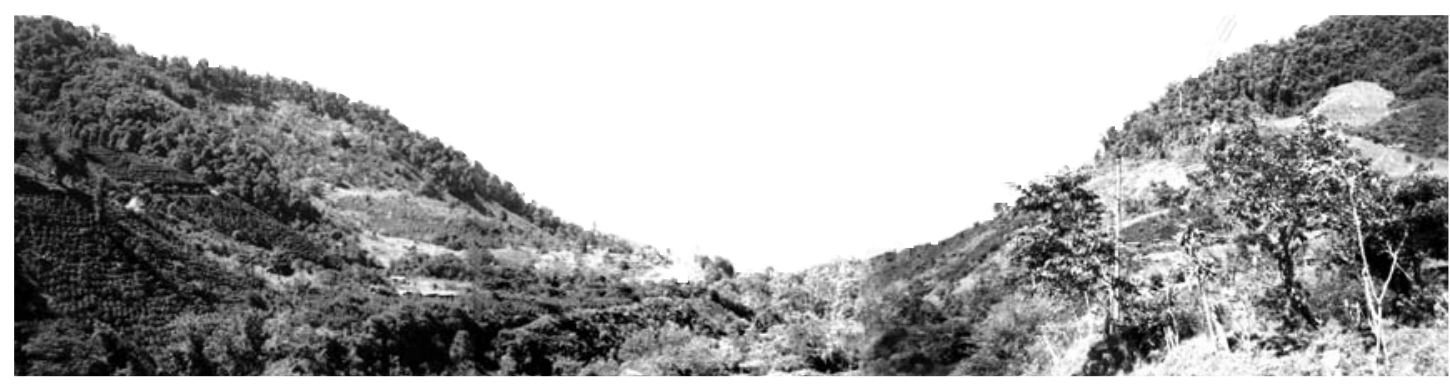

Foto 1. Aspecto general del valle de la cuenca media del río San Pedro entre Cedral y Santa Teresa. Notese la forma en "U" disectada en su fondo por el pequeño valle en "V" del actual río San Pedro.

abanico aluvial, y que es perfectamente observable por una distancia de $5 \mathrm{~km}$ desde la localidad de Santa Teresa hasta aproximadamente $1 \mathrm{~km}$ al norte de la localidad de Cajón. Este valle muestra además de una sección transversal típica en "U", taludes laterales verticales y paralelos, con depósitos megalíticos en su fondo. Llama especialmente la atención el hecho de que el actual río San Pedro, ubicado dentro del antiguo cauce glacial, cambia de curso a unos $100 \mathrm{~m}$ aguas arriba de la localidad de Santa Teresa, y discurre por un nuevo cauce hacia la margen izquierda del valle glacial.En la figura 3 se presenta un par estereográfico en el que se observa la estructura descrita.

La relación de posición relativa entre el abanico de fanglomerados, el valle glacial y el valle fluvial sobreimpuesto, permite la datación relativa de los eventos geológicos del Pleistoceno de esta región de la siguiente manera: 


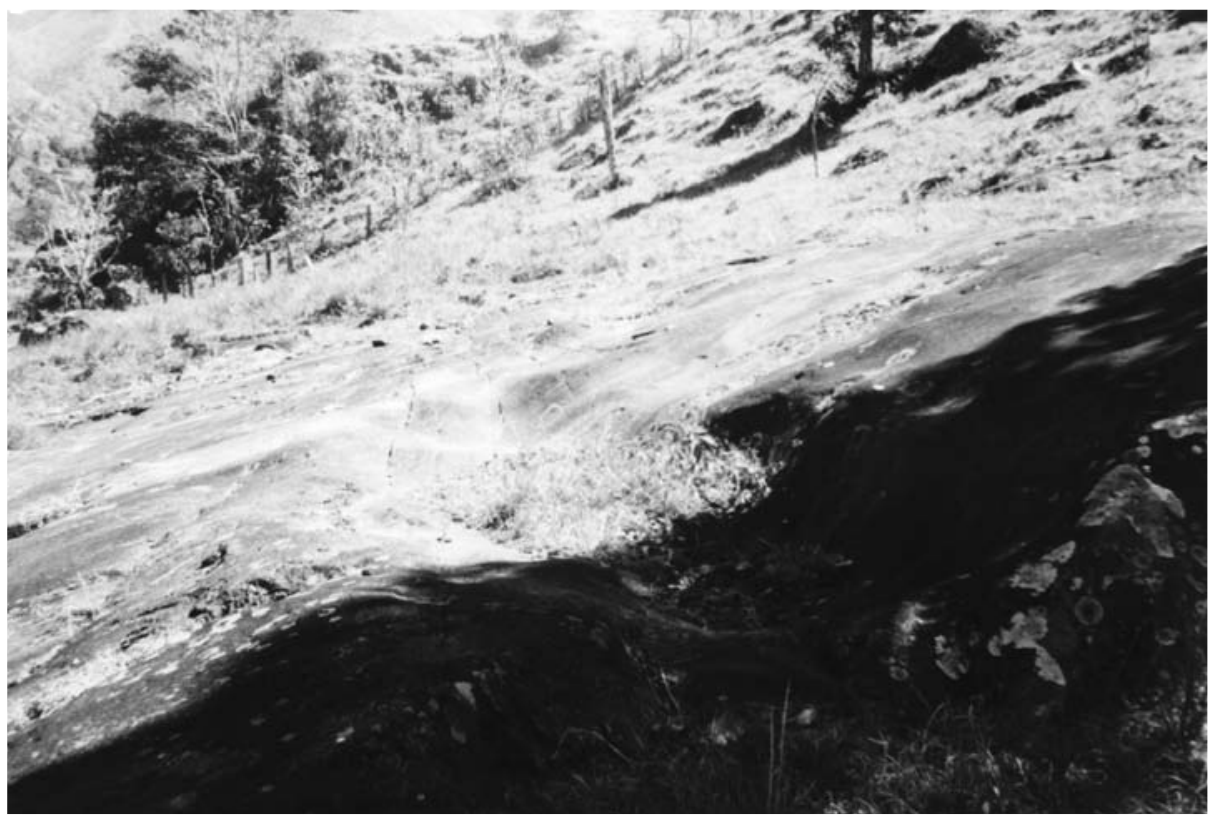

Foto 2. Superficie de roca en la margen izquierda del río San Pedro, aproximadamente a 1 kilómetro aguas arriba de San Jerónimo.

Notese las acanaladuras y estrías producto del desplazamiento del glaciar sobre esta superficie. Esta roca acanalada se localiza a la elevación 1.300 m.s.n.m, y se ubica en el fondo del glaciar San Pedro.

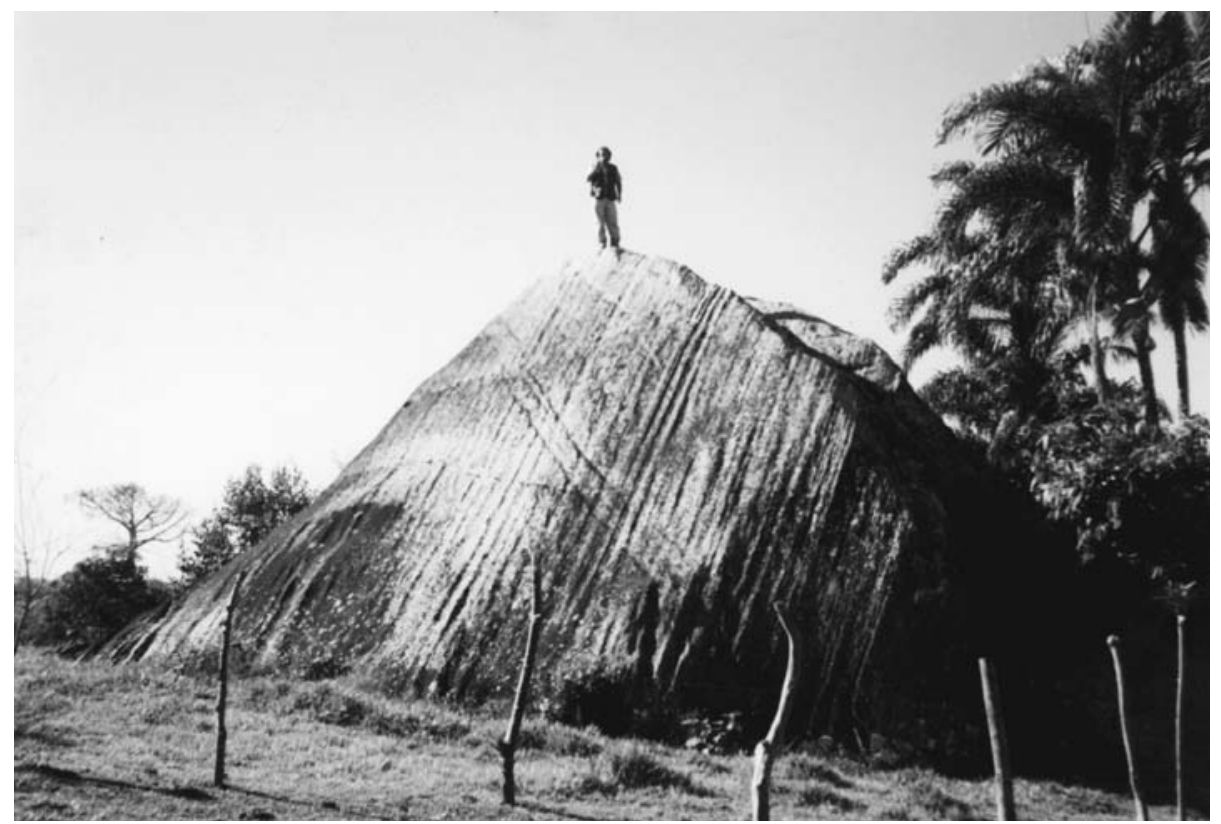

Foto. 3. Bloque magalítico ubicado en las inmediaciones de Laguna a la elevación 680 m.s.n.m, y que corresponde con uno de los bloques y de las morrenas frontales del glaciar San rafael. Esta bloque mide más de $12 \mathrm{~m}$ en su base y muestra estrias y caras planas pulimentadas. Sobre su superficie se encuentran numerosos petroglifos. 

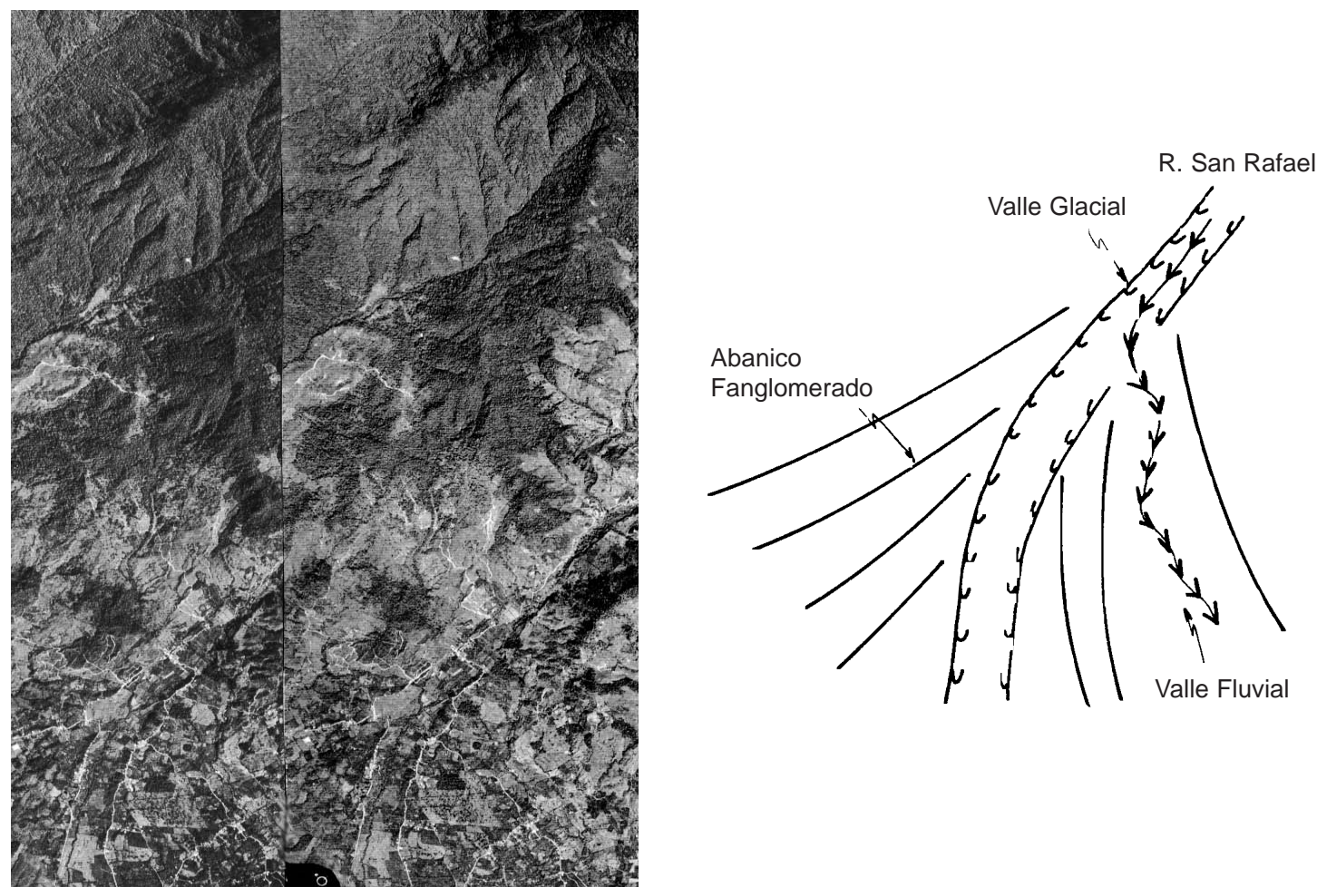

Fig. 3. Fotointerpretación de par estereográfico de la zona de Santa Teresa en la cuenca del río San Pedro. Se observa como la erosión fluvial de este río produjo un cambio de curso hacia la margen izquierda de manera que el valle glacial ubicado hacia aguas abajo quedo "fosilizado". Dentro de este valle es posible observar depósitos de megalitos con distribución errática característicos del modelado glacial.

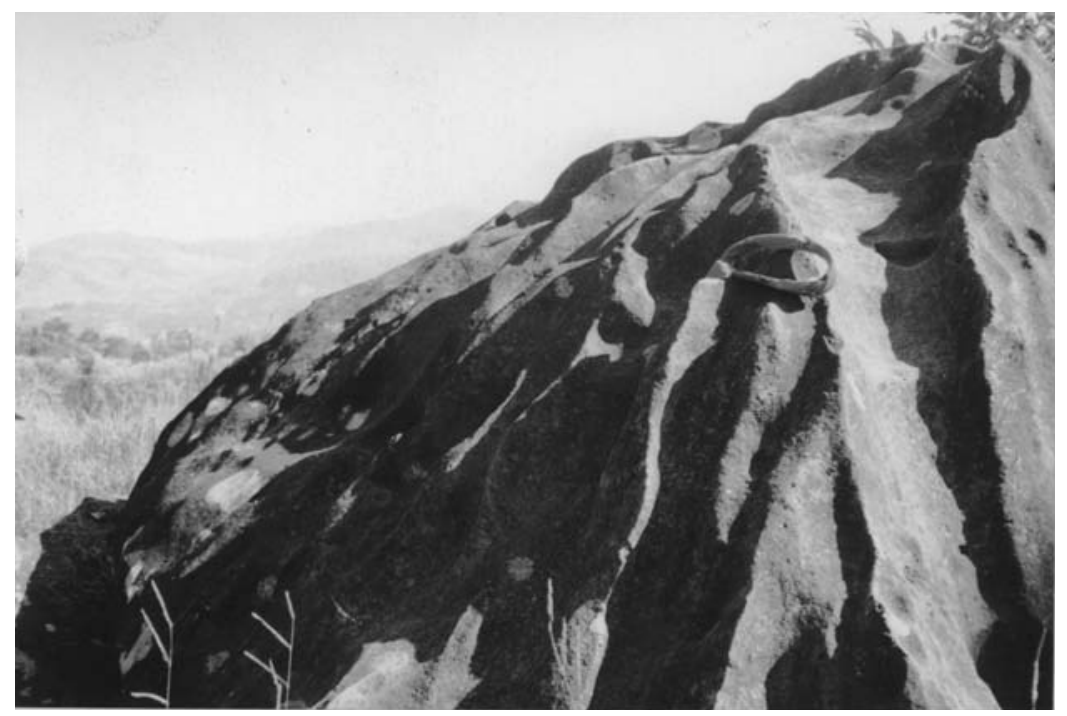

Foto. 4. Megalito ubicado cerca de la localidad de la Guaria en la cuenca del río San Rafael. Nótese la textura acanalada y estriada de la superficie de la roca. 


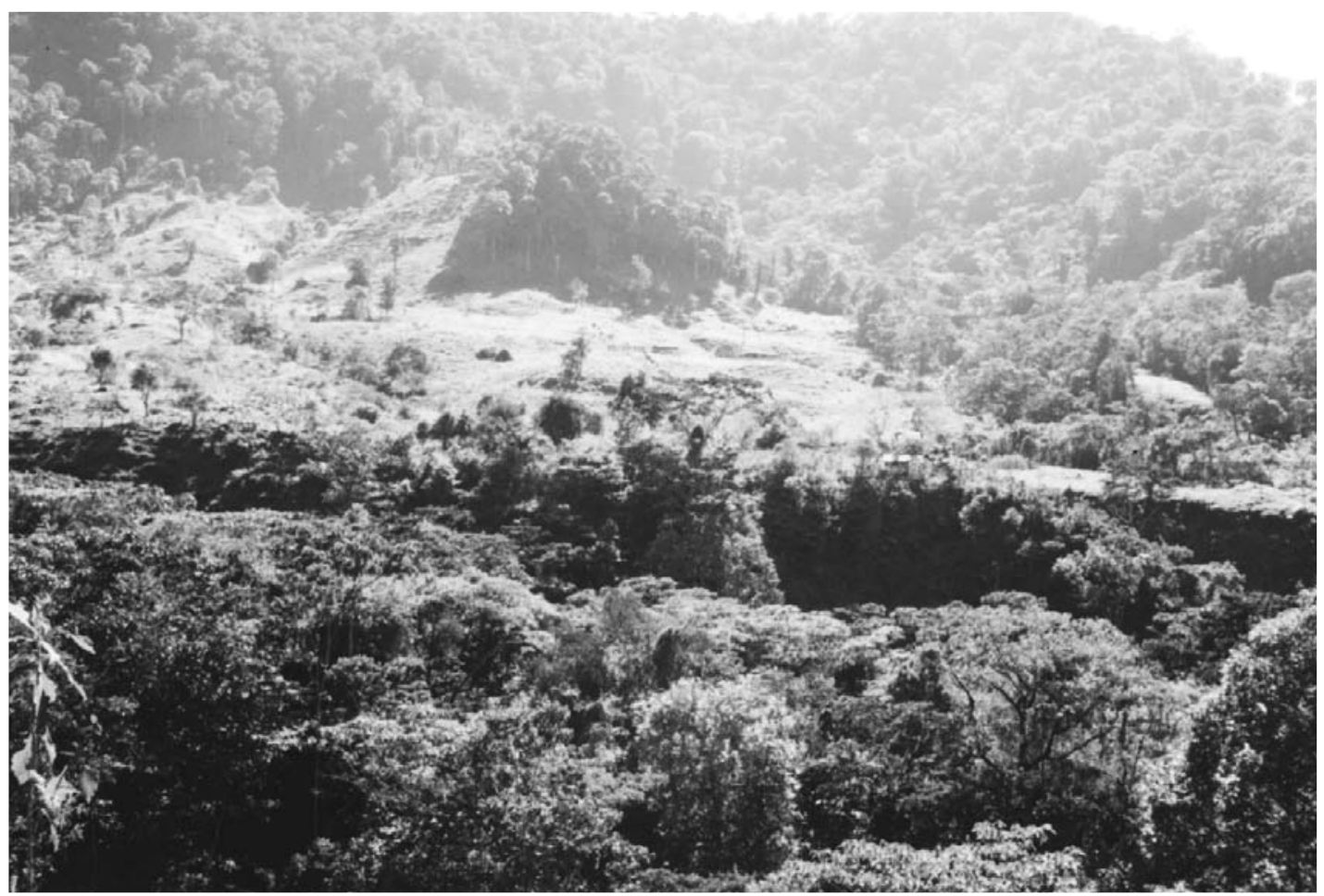

Foto. 5. Vista panorámica del valle del río San Pedro en el sector de Cedral. En este valle se observa una morfología de tipo "artesa" o "circo" de fondo plano con sección transversal en "U", disectada en su centro por el actual valle fluvial de San Pedro con una profunda sección en "U".. La "planice" que se observa en la fotografía está conformada por depósitos de megalitos con distribución granulométrica errática.

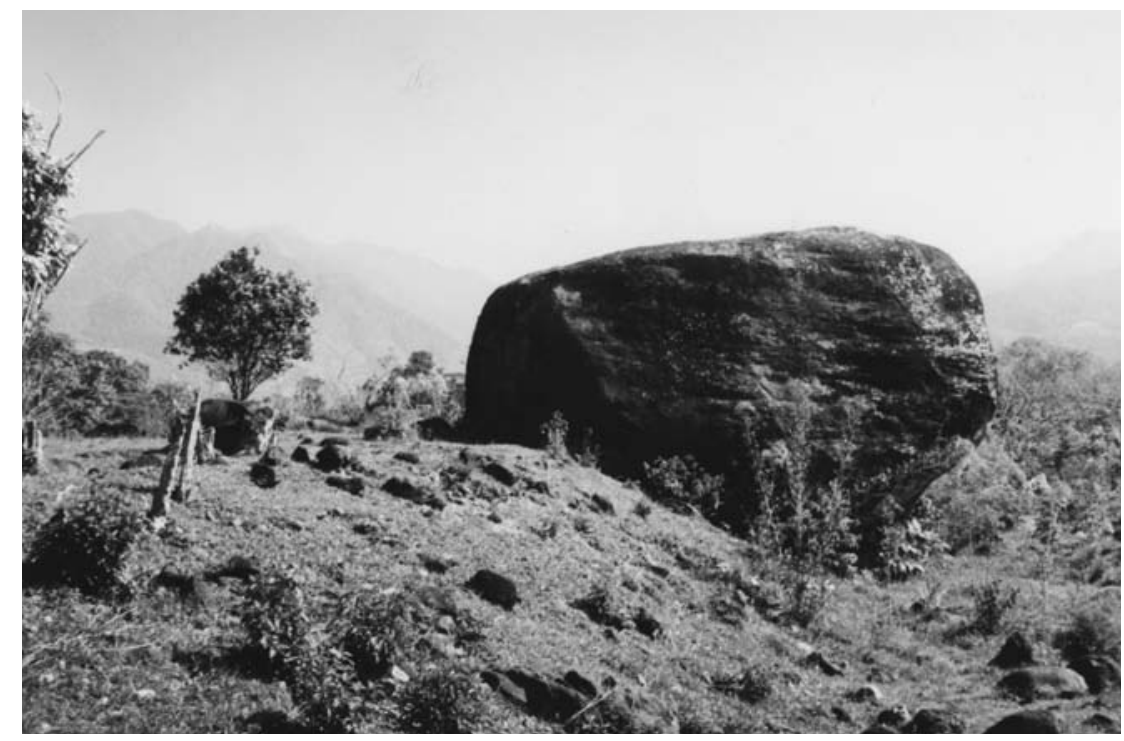

Foto. 6. Bloque de dimensiones megalíticas en una posible morrena frontal de glaciar de San Rafael. Este bloque mide más de $15 \mathrm{~m}$ de artista, muestra estrias, y su superficie presenta abundantes petroglifos. El peso estimado de este bloque supera las 1.500 toneladas. 
- Hacia finales del Terciario, posiblemente entre el Mioceno y el Plioceno, se produce el levantamiento intenso de la actual cordillera de Talamanca, lo cual a su vez originó su erosión masiva conforme se levantaba la cordillera.

- La erosión de la cordillera produjo enormes flujos de sedimentos hacia el Valle del General, los cuales se emplazaron en forma de abanicos que alcanzan los $10 \mathrm{~km}$ de extensión en su frente, más de $6 \mathrm{~km}$ en su eje longitudinal, y espesores que superan los $100 \mathrm{~m}$ en su centro. Uno de estos abanicos se observa actualmente en la zona comprendida entre los ríos Peñas Blancas y Unión, y se extiende desde la actual carretera Interamericana hasta el inicio de la cordillera de Talamanca (Fig.2). El volúmen estimado de este abanico supera el km3.
- La edad de formación de estos abanicos comprende los períodos Plioceno y Pleistoceno, y posiblemente se trate de muchos eventos de emplazamiento masivo combinados y/o acelerados por los deshielos de las tres primeras glaciaciones del Pleistoceno.

- Hacia finales del Pleistoceno, y posiblemente durante la glaciación de Wisconsin (correlacionada con los períodos Wurm II y III, según Meléndez-Fuster, 1975), que tuvo su climax hace unos 50.000 años y se extendió hasta hace unos 10.000 años, cuando se inició el período interglacial actual, se emplazaron dentro de los cauces actuales de los ríos Peñas Blancas, San Pedro y San Rafael, glaciares tipo "lengua" que alcanzaron extensiones de más de $10 \mathrm{~km}$. Algunas de esta lenguas glaciares se extendieron hasta elevaciones

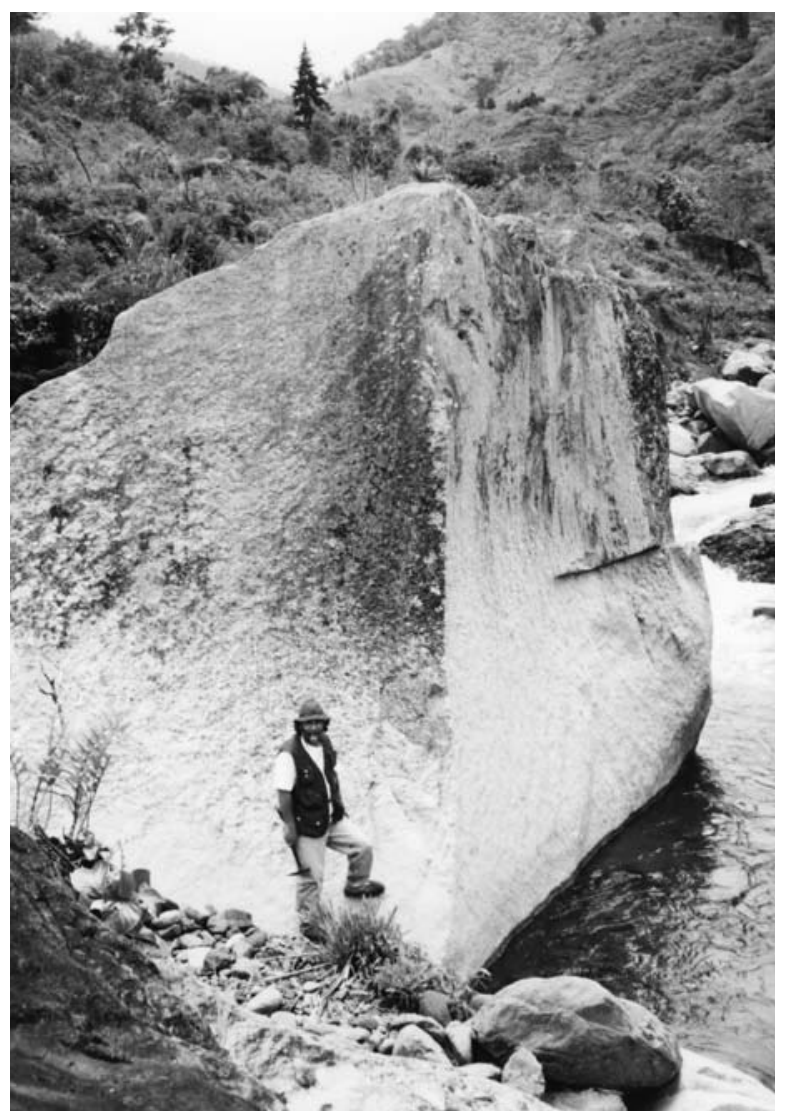

Foto 7. Megabloque de Granodiorita con forma casi cúbica con más de $5 \mathrm{~m}$ de arista, caras planas, pulidas y octogonales. Este tipo de bloques, abundantes en los depósitos cercanos al río San Rafael, pudieron ser acarreados por glaciares tipo lengua desde la cordillera. 
menores a los 1000 m.s.n.m, originando los depósitos de bloques erráticos megalíticos y los depósitos de morrenas que se observan actualmente en la zona del piedemonte de la cordillera.

- El glaciar de San Pedro se emplazó sobre los abanicos aluviales preexistentes, tal como se observa en el paleovalle glacial ubicado entre Santa Teresa y Cajón, dentro del que discurre actualmente la quebrada Cajón. Este hecho evidencia la antecedencia de la depositación de los abanicos con respecto al emplazamiento de la lengua del glaciar de San Pedro.

- Durante el período comprendido entre el final de la glaciación de Wisconsin y la actualidad, la erosión fluvial produjo el modelado particular que muestran los cauces actuales de los ríos San Pedro y San Rafael, en donde se observa valles fluviales en "V" cortando los extensos valles en "U". El río San Pedro, erosionó los depósitos laterales sobre su margen izquierda a unos $100 \mathrm{~m}$

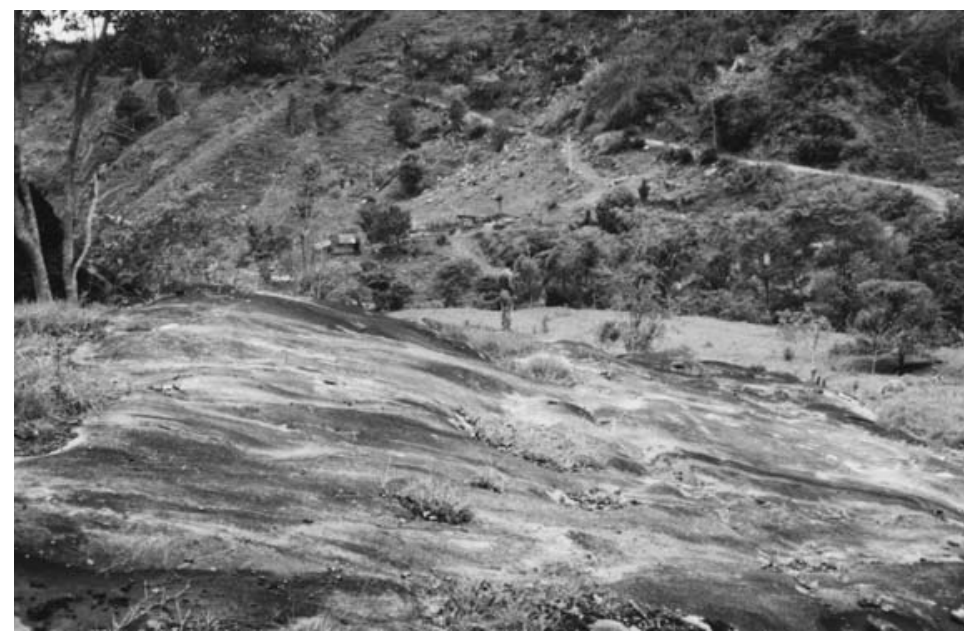

Foto. 8. Superficie de erosión glaciar sobre la margen izquierda del río San Rafael, aproximadamente a $1.5 \mathrm{~km}$ aguas arriba de San Jerónimo. Se observa sobre esta roca los efectos de la erosión glacial de fondo en forma de estrias y acanaladuras paralelas al curso actual del río.

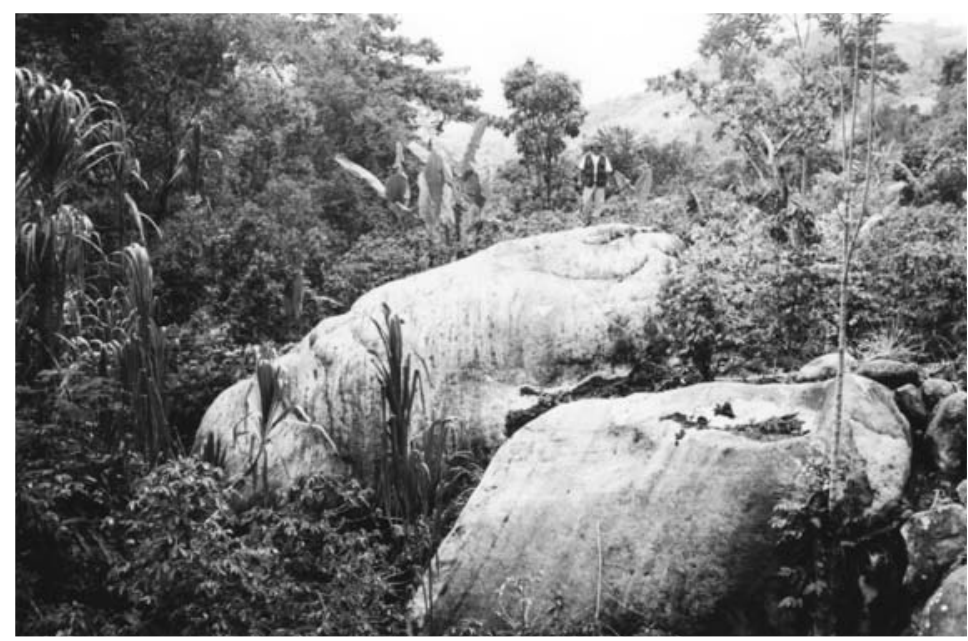

Foto. 9. Megabloques estriados con caras pulidas presentes en los depósitos periglaciares de la margen derecha del río San Rafael. Este tipo de depósitos es característico de la morfología glaciar que conforma el relieve reciente del área investigada. 


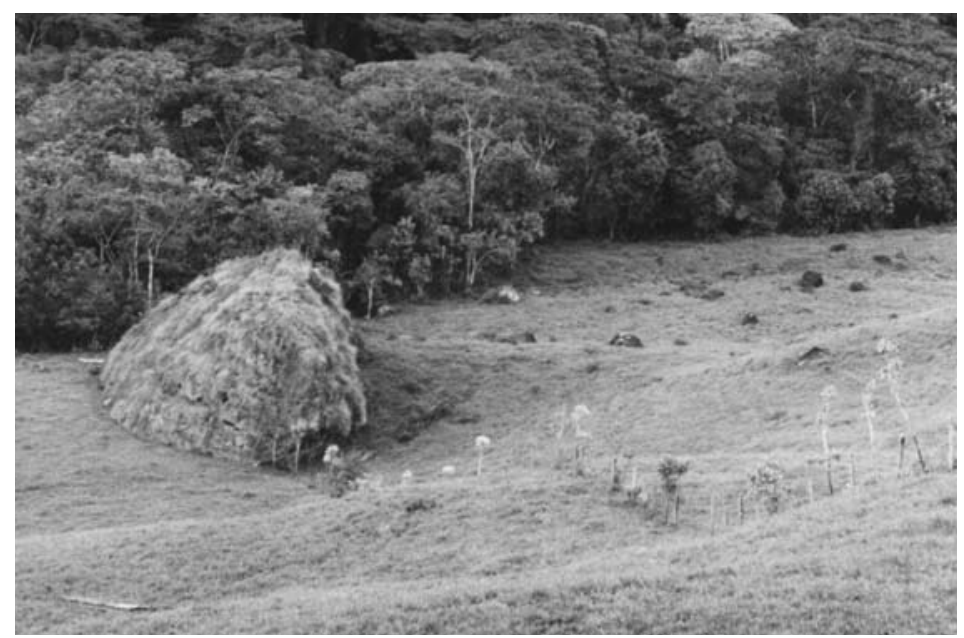

Foto. 10. Depósito de origen glacial con megabloques en posibles morrenas frontales. Este bloque mide más de $15 \mathrm{~m}$ en la base aguas arriba se presentan acumulaciones erráticas de bloques. Este depósitos se ubica sobre la margen izquierda del río Peñas Blanquitas, a una elevación de 980 m.s.n.m.

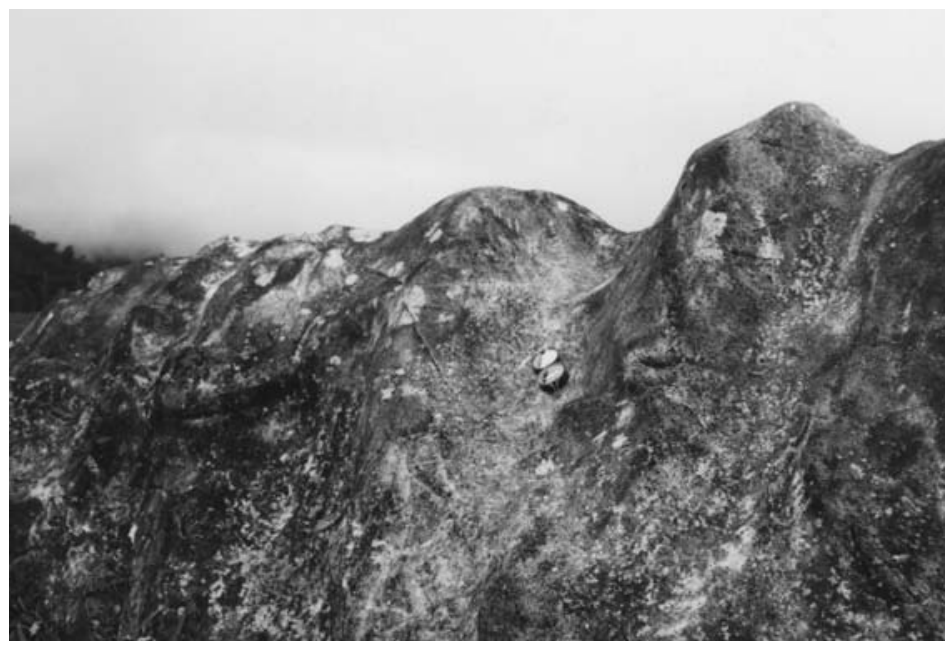

Foto 11: Detalle de la textura acanalada y estriada que presenta la mayoría de los megabloques. Los canales tienen una amplitud de "onda" cerca de $15 \mathrm{~cm}$.

aguas arriba de Santa Teresa, y produjo un cambio de curso que a la postre contribuyó a mantener las características originales del valle glaciar aguas abajo de este sitio.

Las evidencias que se describen en este trabajo permiten incluir a la acción de glaciares tipo lengua como parte de los fenómenos morfogenéticos que conformaron la morfología de la región de piedemonte de la cordillera de Talamanca, en su sector occidental, tanto desde las elevaciones de más de
3000 m.s.n.m descritas por Weyl (1957), como a elevaciones menores que 1000 m.s.n.m. De acuerdo con el Dr. Luis Guillermo Brenes (comunicación verbal), la causa posible de que las lenguas glaciares descendieran hasta elevaciones menores que 1000 m.s.n.m en el flanco occidental de la cordillera se relaciona con el hecho de que este sector se localiza a "umbria" de manera que no está expuesta a la radiación solar durante muchas horas al día. 


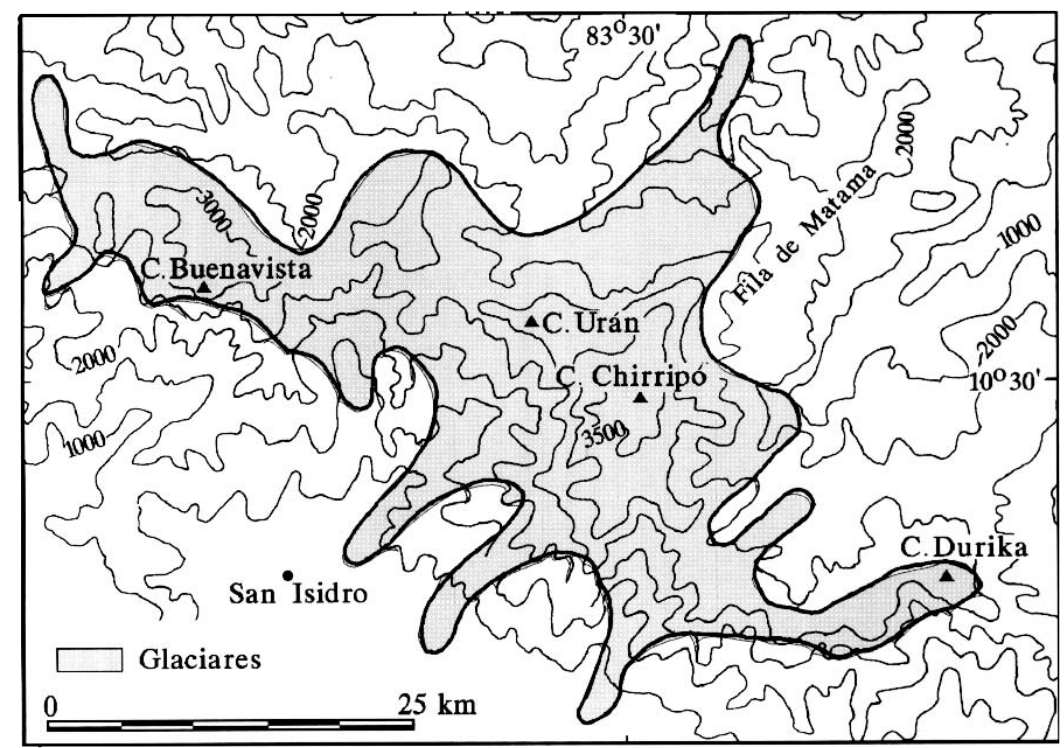

Fig. 4. Reconstrucción del posible aspecto de la cordillera de Talamanca, en el sector del macizo del Chirripó, y de las lenguas glaciares que alcanzaron al Valle del General, durante el climax de la glaciación de Wisconsin, hace unos 50000 años.

\section{CONCLUSIONES}

Con base en las observaciones de campo se ha interpretado la existencia de glaciares tipo lengua, los cuales se emplazaron dentro de los cauces actuales de los ríos Peñas Blancas, San Pedro y San Rafael, ubicados sobre el flanco suroeste de la cordillera de Talamanca, en el sector del macizo del Chirripó.

Estos glaciares se extendieron por más de $10 \mathrm{~km}$ desde las partes altas de la cordillera hasta la región del piedemonte, y su frente alcanzó elevaciones de menos de 1000 m.s.n.m actual.

Aunque este no pretende ser un estudio exahustivo de las características del modelado glaciar del Pleistoceno tardío en el Valle del General, se ha tratado de reconstruir el aspecto de esta parte de la cordillera durante el climax del período glacial que hemos correlacionado con la glaciación de Wisconsin y cuyo climax se produjo hace unos 50.000 años. En la figura 4 se muestra la reconstrucción de los glaciares tipo lengua que se extendieron desde la cordillera hasta el Valle del General. Posiblemente existió este tipo de glaciares dentro de las actuales cuencas de los ríos Volcán y Ceibo ubicadas hacia el este de la zona investigada, y que pudieron originarse en el flanco suroeste del Cerro Durika.

\section{AGRADECIMIENTOS}

A los Drs. Carlos Meléndez Chaverri y Luis Guillermo Brenes Quesada por su gentileza en la revisión y corrección del manuscrito, a Silvia Meléndez Dobles por su valiosa ayuda en la preparación de las láminas y a Rodolfo Espinach Leitón por su asistencia en los trabajos de campo.

\section{BIBLIOGRAFÍA}

MELÉNDEZ, B \& FUSTER,J., 1975: Geología. - Editorial Paraninfo, Madrid España.

MORA, S. 1979: Proyecto Hidroeléctrico de Boruca. Estudio Geológico Regional. - Instituto Costarricense de Electricidad, San José.

SPRECHMNAN, P., 1984: Manual de Geología de Costa Rica. - Editorial Universidad de Costa Rica. San José.

WEYL, R., 1957: Excursiones geológicas en Costa Rica: vestigios de los glaciares del Pleistoceno. - Inst. Geogr. Nac., Inf. Trimestr. Enero-Marzo, 50 págs. San José, Costa Rica. 